\title{
MATHEMATICAL MODEl OF AN ANTHROPOMORPHIC ROBOTS ARM
}

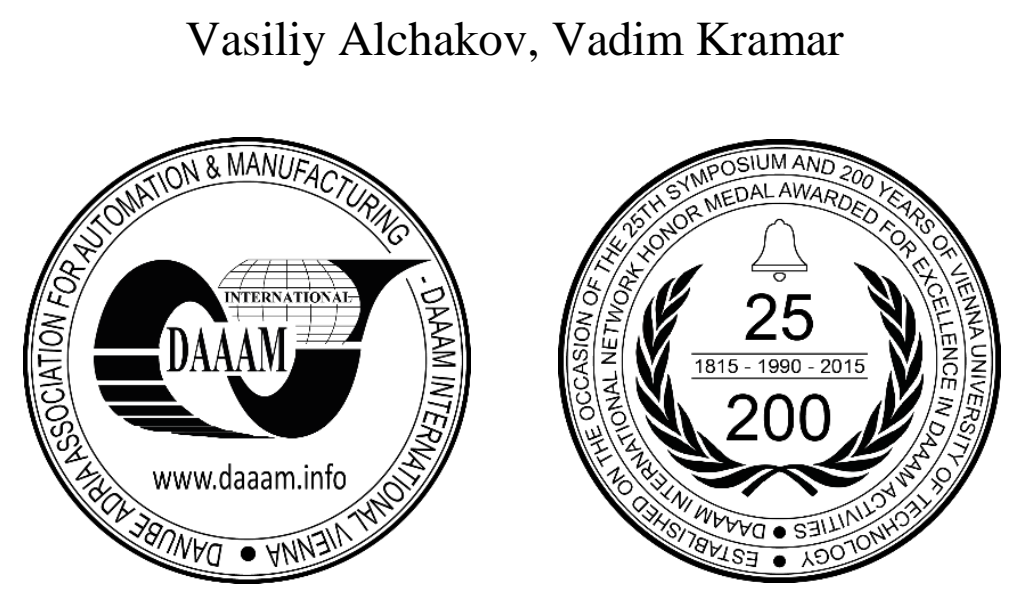

This Publication has to be referred as: Alchakov, V[asiliy] \& Kramar, V[adim] (2018). Mathematical Model of an Anthropomorphic Robots Arm, Proceedings of the 29th DAAAM International Symposium, pp.0095-0101, B. Katalinic (Ed.), Published by DAAAM International, ISBN 978-3-902734-20-4, ISSN 1726-9679, Vienna, Austria DOI: $10.2507 / 29$ th.daaam.proceedings.013

\begin{abstract}
One of the main tasks of developers of robotic systems based on humanoid robots is to create robotic systems with autonomous and developed skills of fine motor manipulator arms. Effective skills for manipulating robots in unstructured environments are fundamental to carrying out manipulation tasks and to ensure proper and safe interaction between people and robots. The main purpose of this work was to develop a mathematical model of the arm of an anthropomorphic robot, which can actually be divided into two sub models: the model of the elbow and shoulder joints and the model of the hand. The direct problem of kinematics was solved - the position and orientation of the manipulator's grasp with respect to the absolute coordinate system were determined from the known vector of the attached angles and by the number of degrees of freedom. As the prototype, the authors used an anthropomorphic robot model SAR-400.
\end{abstract}

Keywords: anthropomorphic robot; manipulator arms; mathematical model; robot SAR-400; the Denavit-Hartenberg method; kinematics problems

\section{Introduction}

The arm is one of the most complex elements of the human body, which participates in information and executive processes that ensure human life activity. The arm is used to capture objects. Man's hands can, in the overwhelming majority, adapt to objects of any shape in order to accomplish the grab. Therefore, one of the main tasks for developers of anthromorphic robotic systems is to create robotic systems with autonomous and developed skills of fine motor skills of arms. Effective skills for manipulating robots in unstructured environments are fundamental to carrying out manipulation tasks and to ensure proper and safe interaction between people and robots [1], [2]. Therefore, for the development of such systems, it is necessary to have a complete mathematical model of the manipulator, on the basis of which it is possible to program the system and build control laws. Despite a large number of studies in this field [3], [4], [5], [6] due to the continuous improvement of robotic systems, further research is needed. The main purpose of this work was to develop a mathematical model of the arm of an anthropomorphic robot with help of solving the direct problem of kinematics - the position and orientation of the manipulator's grasp with respect to the absolute coordinate system were determined from the known vector of the attached angles and by the number of degrees of freedom. The article deals with the construction of a mathematical model for the arm of the robot SAR-400 (see fig. 1 and 2). 


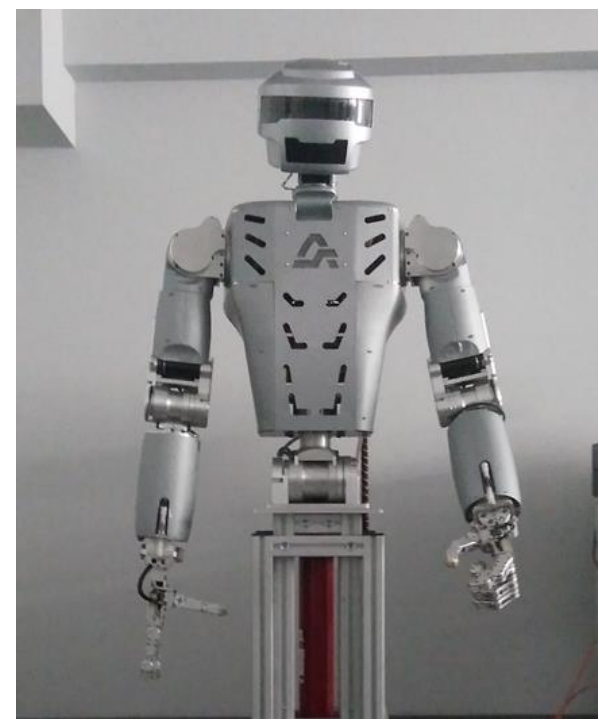

Fig. 1. Robot SAR-400

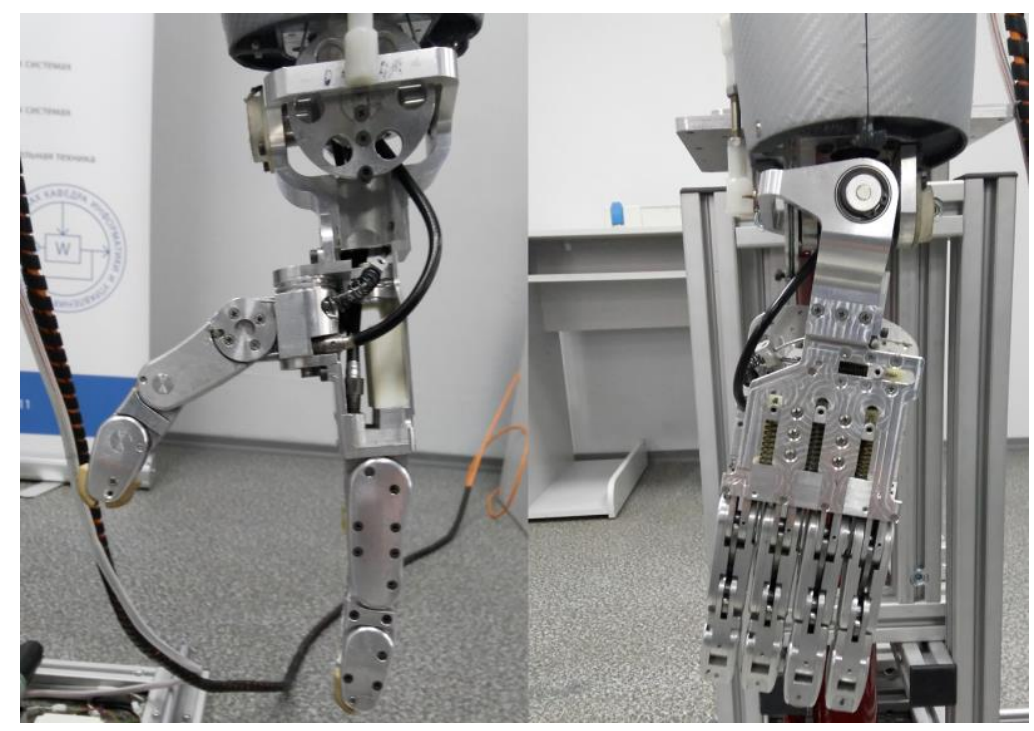

Fig. 2. General view of the SAR-400 arm

The SAR-400 arm differs from the actual human arm. The difference lies in the as follows: the ring finger and the little finger are combined; control of individual finger joints is impossible. The movements of the fingers are controlled by a single motor. For simplicity, it is assumed that the angles that describe the rotation of the finger joints are equal to each other. Angles have limit values.

\section{The Denavit-Hartenberg describing the neighboring links of the manipulator}

\subsection{The Denavit-Hartenberg Method}

To describe the rotational and translational relations between adjacent links of manipulator, Denavit and Hartenberg proposed a matrix method for sequentially constructing coordinate systems associated with each link in the kinematic chain. The meaning of the representation of Denavit-Hartenberg consists in the formation of a homogeneous transformation matrix having a dimension of $4 \times 4$ and describing the position of the coordinate system of each link relative to the coordinate system of the previous link. This makes it possible to successively convert the coordinates of the grip of the manipulator from the frame of reference connected with the last link to the reference frame, which is an inertial coordinate system for the dynamical system under consideration [7], [8], [9].

Each coordinate system is formed on the basis of the following three rules:

1. The $z_{i-1}$ axis is directed along the axis of the $i-t h$ junction;

2. The $x_{i}$ axis is perpendicular to the $z_{i-1}$ axis and is directed away from it;

3. The $y_{i}$ axis completes the $x_{i}, z_{i}$ axes to the right Cartesian coordinate system.

The representation of the Denavit-Hartenberg solid links depends on the four geometric parameters corresponding to each link. These four parameters completely describe any rotational or translational motion and are defined as follows:

- $\theta_{i}$ is the adjoined angle, on which it is necessary to rotate the $x_{i-1}$ axis around the $z_{i-1}$, so that it becomes codirectional with the $x_{i}$ axis;

- $d_{i}$ is the distance between the intersection of the $z_{i-1}$ axis with the xi axis and the beginning of the $(i-1)-$ th coordinate system, measured along the $z_{i-1}$ axis;

- $r_{i}$ is the distance between the intersection of the $z_{i-1}$ axis with the $x_{i}$ axis and the beginning of the $i$ - th coordinate system, measured along the $x_{i}$ axis;

- $\alpha_{i}$ - angular displacement - angle, on which it is necessary to turn the $z_{i-1}$ axis about the axis $x_{i}$, so that it becomes co-directional with the $z_{i}$-axis.

For the robot SAR-400 for rotational joints, the parameters $d_{i}, r_{i}$ and $\alpha_{i}$ are constant. $\theta_{i}$ is a variable that varies as the $i$ - th link rotates relative to $(i-1)-$ th. 


\subsection{Algorithm for the formation of coordinate systems of links}

The proposed algorithm for each link of the manipulator with $n$ degrees of freedom forms an orthonormal coordinate system. Coordinate systems are numbered in ascending order from the base to the grip of the manipulator. The mutual arrangement of neighboring links is described by a $4 \times 4$ homogeneous transformation matrix.

Here is the algorithm for forming the coordinate system of the links:

a) The formation of a basic coordinate system. Form the right orthonormal coordinate system $\left(x_{0}, y_{0}, z_{0}\right)$ associated with base. The $z_{0}$ axis is directed along the axis of the 1-st joint to the arm of the manipulator. Axes $x_{0}$ and $y_{0}$ are chosen arbitrarily under the condition of their perpendicularity axis $z_{0}$.

b) For all $i(i=1,2, \ldots n-1)$ follow the steps c) $-\mathrm{e})$.

c) Formation of the articulation axes. We direct the $z_{i}$ axis along the axis of motion (rotational or translational) $(i+1)$-th junction.

d) Formation of the origin of the $i$-th coordinate system. Arrange the beginning of $i$-th coordinate systems at the intersection of the axes $z_{i}$ and $z_{i-1}$ or at the intersection of the common normal to the axes $z_{i}$ and $z_{i-1}$ with the $z_{i}-$ axis.

e) Formation of the $x_{i}$ axis. We choose the unit vector $x_{i}$ along the common perpendicular to the axes $z_{i-1}$ and $z_{i}$ if they are parallel.

f) Formation of the $y_{i}$ axis. We choose $y_{i}$ so as to get a right-handed coordinate system;

i) Formation the grip of the coordinate system. The $n$-th junction is rotational. We form the $z_{n}$ axis, pointing it along the $z_{i-1}$ axis and from the robot. We choose the $x_{n}$ axis so that it is perpendicular to the axes $z_{n-1}$ and $z_{n}$.

j) Determination of link and joint parameters. For each $i(i=1,2, \ldots n)$ follow steps $\mathrm{k})-\mathrm{n})$;

k) Definition of $d_{i}$. The distance di is the distance from the origin of the $(i-1)$ - th coordinate system to the intersection of the $z_{i-1}$ axis with the $x_{i}$ - axis and the origin of the $i$-th coordinate system, measured along the $z_{i-1}$ axis. If the $i$-th connection is translational, then $d_{i}$ - the attached variable.

1) The definition of $r_{i}$ is the distance between the intersection of the $z_{i-1}$ axis with the $x_{i}$ axis and the beginning of the $i$-th coordinate system, measured along the $x_{i}$-axis.

m) The definition of $\theta_{i}$ - the angle of rotation of the $x_{i-1}$ axis about the $z_{i-1}$ axis, so that it becomes is aligned with the $x_{i}$ axis. If the $i$-th junction is rotational, then $\theta_{i}$-attached variable.

n) The definition of $\alpha_{i}$ - the angle of rotation of the $z_{i-1}$ axis about the $x_{i}$ axis, so that it becomes is aligned with the $z_{i}$ axis.

After the construction of coordinates for all links it is possible to construct homogeneous transformation matrices connecting the $i$-th and $(i-1)$ - th systems coordinates:

$$
A_{i}=\left[\begin{array}{cccc}
\cos \theta_{i} & -\sin \theta_{i} \cos \alpha_{i} & \sin \theta_{i} \sin \alpha_{i} & r_{i} \cos \theta_{i} \\
\sin \theta_{i} & \cos \theta_{i} \cos \alpha_{i} & -\cos \theta_{i} \sin \alpha_{i} & r_{i} \sin \theta_{i} \\
0 & \sin \alpha_{i} & \cos \alpha_{i} & d_{i} \\
0 & 0 & 0 & 1
\end{array}\right]
$$

The resulting transformation matrices must be multiplied, resulting in a matrix of transition to the original coordinates. Using the matrix, we can relate the homogeneous coordinates $p_{i}$ of the point $p$ with respect to the $i$ - th coordinate system (the point $p$ is at rest in the $i$ - th coordinate system) with one-sided coordinates of this point with respect to the $(i-1)$ - th system, connected with the $(i-1)$ - th link. This relationship is established by the equality:

$$
p_{i}=A_{i} p_{i-1}
$$

As mentioned above, the approach based on the Denavit-Hartenberg method was used to solve the task of describing the position of the SAR-400 arm. The difference from the original Denavit-Hartenberg method lies in the fact that for a given problem the parameter $\alpha$ of one of the links was adopted by variables, and one of the parameters $\theta$ was adopted as a constant. Since the Denavit-Hartenberg method describes the position of one end point of the manipulator, then in order to get the model of the SAR-400 arm it is necessary to solve the kinematics problem for each of the fingers of the arm. 


\section{Solving the kinematics problems}

\subsection{The Direct Problem of Kinematics}

The direct problem of kinematics consists in finding the coordinates of the end of the kinematic chain, with known lengths of links and the angles between them. The SAR-400 arm has no links that change its length, therefore all lengths in describing the kinematic chain will be constant. When solving kinematics problems, it is necessary to take into account the limitations which arise from the peculiarities of the structure of SAR-400. The limitations that have arisen due to the peculiarity of the problem include the same value of the angles that describe the turn of the robot arm, for each of the fingers. The links of the four fingers (index, middle, nameless and little finger) have the same kinematic chains (see fig.3). The only difference in their structure is the displacement of the finger relative to the center of the arm.

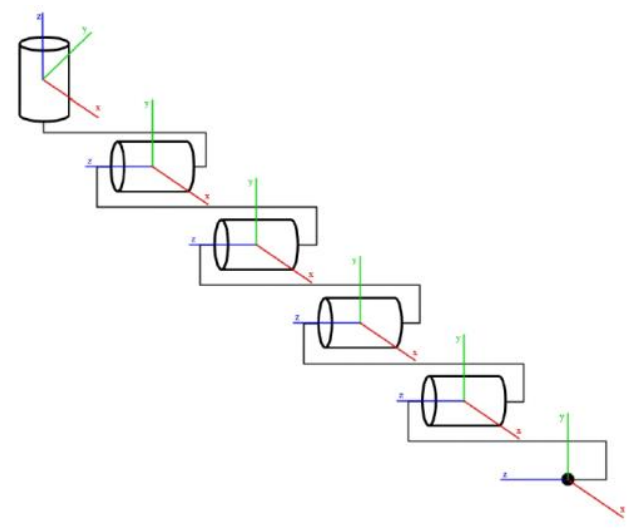

Fig. 3. Kinematic chain of the little finger, ring finger, middle finger and index finger

The structure of the thumb differs from the structure of the other fingers, so its kinematic chain will be different (see fig.4).

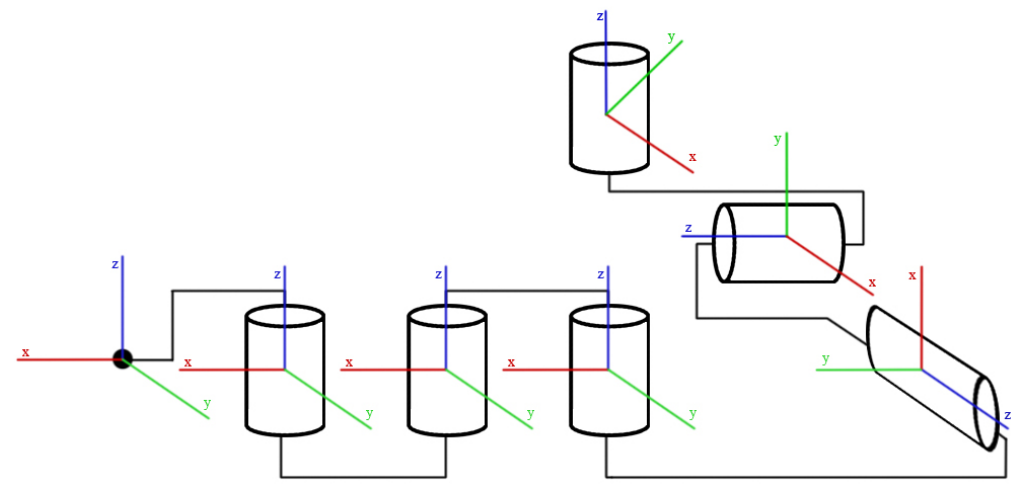

Fig. 4. Kinematic chain of the thumb

We give the solution of the direct kinematics problem for the little finger. For the other three fingers, the problem is solved similarly, taking into account the value of the variable $d$. Based on the kinematic chain of the finger, Denavit Hartenberg parameters, listed in the table 1.

\begin{tabular}{|c|c|c|c|c|}
\hline № link & $\mathbf{d , ~} \mathbf{m}$ & $\mathbf{r , ~} \mathbf{m}$ & $\boldsymbol{\theta}$ & $\boldsymbol{\alpha}$ \\
\hline 1 & 0 & 0 & $\theta_{1}$ & 90 \\
\hline 2 & 0,0319 & 0,158 & $\theta_{2}$ & 0 \\
\hline 3 & 0 & 0,038 & $\theta_{3}$ & 0 \\
\hline 4 & 0 & 0,028 & $\theta_{4}$ & 0 \\
\hline 5 & 0 & 0,022 & $\theta_{5}$ & 0 \\
\hline
\end{tabular}

Table 1. Denavit-Hartenberg parameters for the little finger

From relation (1) we obtain transformation matrices for each of the links. 


$$
\begin{aligned}
A_{1} & =\left[\begin{array}{cccc}
\cos \theta_{1} & 0 & \sin \theta_{1} & 0 \\
\sin \theta_{1} & 0 & -\cos \theta_{1} & 0 \\
0 & 1 & 0 & 0 \\
0 & 0 & 0 & 1
\end{array}\right], A_{2}=\left[\begin{array}{cccc}
\cos \theta_{2} & -\sin \theta_{2} & 0 & 0,158 \cos \theta_{2} \\
\sin \theta_{2} & \cos \theta_{2} & 0 & 0,158 \sin \theta_{2} \\
0 & 0 & 1 & 0,0319 \\
0 & 0 & 0 & 1
\end{array}\right], \\
A_{3} & =\left[\begin{array}{cccc}
\cos \theta_{3} & -\sin \theta_{3} & 0 & 0,038 \cos \theta_{3} \\
\sin \theta_{3} & \cos \theta_{3} & 0 & 0,038 \sin \theta_{3} \\
0 & 0 & 1 & 0 \\
0 & 0 & 0 & 1
\end{array}\right], A_{4}=\left[\begin{array}{cccc}
\cos \theta_{4} & -\sin \theta_{4} & 0 & 0,028 \cos \theta_{4} \\
\sin \theta_{4} & \cos \theta_{4} & 0 & 0,028 \sin \theta_{4} \\
0 & 0 & 1 & 0 \\
0 & 0 & 0 & 1
\end{array}\right], \\
A_{5} & =\left[\begin{array}{cccc}
\cos \theta_{5} & -\sin \theta_{5} & 0 & 0,022 \cos \theta_{5} \\
\sin \theta_{5} & \cos \theta_{5} & 0 & 0,022 \sin \theta_{5} \\
0 & 0 & 1 & 0 \\
0 & 0 & 0 & 1
\end{array}\right] .
\end{aligned}
$$

Multiplying the transformation matrices and obtaining the resulting matrix we obtain formulas describing the position of the finger in space.

$$
\begin{aligned}
p_{x}= & 0,0158 c_{1} c_{2}-0,028 s_{4}\left(c_{1} c_{2} s_{3}+c_{1} c_{3} s_{2}\right)-0,022 s_{5}\left[c_{4}\left(c_{1} c_{2} s_{3}+c_{1} c_{3} s_{2}\right)-s_{4}\left(c_{1} s_{2} s_{3}-c_{1} c_{2} c_{3}\right)\right]- \\
& -0,028 c_{4}\left(c_{1} s_{2} s_{3}-c_{1} c_{2} s_{3}\right)+0,0319 s_{1}-0,022 c_{5}\left[s_{4}\left(c_{1} c_{2} s_{3}+c_{1} c_{3} s_{2}\right)+c_{4}\left(c_{1} s_{2} s_{3}-c_{1} c_{2} c_{3}\right)\right]- \\
& -0,038 c_{1} s_{2} s_{3}+0,038 c_{1} c_{2} c_{3}, \\
p_{y}= & 0,0158 c_{2} s_{1}-0,028 s_{4}\left(c_{2} s_{1} s_{3}+c_{3} s_{1} s_{2}\right)-0,022 s_{5}\left[c_{4}\left(c_{2} s_{1} s_{3}+c_{3} s_{1} s_{2}\right)-s_{4}\left(s_{1} s_{2} s_{3}-c_{2} c_{3} s_{1}\right)\right]- \\
& -0,028 c_{4}\left(s_{1} s_{2} s_{3}-c_{2} c_{3} s_{1}\right)-0,0319 c_{1}-0,022 c_{5}\left[s_{4}\left(c_{2} s_{1} s_{3}+c_{3} s_{1} s_{2}\right)+c_{4}\left(s_{1} s_{2} s_{3}-c_{2} c_{3} s_{1}\right)\right]- \\
& -0,038 s_{1} s_{2} s_{3}+0,038 c_{2} c_{3} s_{1}, \\
p_{z}= & 0,0158 s_{2}+0,038 c_{2} s_{3}+0,038 c_{3} s_{2}+0,028 c_{4}\left(c_{2} s_{3}+c_{3} s_{2}\right)+0,028 s_{4}\left(c_{2} c_{3}-s_{2} s_{3}\right)+ \\
& +0,022 c_{5}\left[c_{4}\left(c_{2} s_{3}+c_{3} s_{2}\right)+s_{4}\left(c_{2} c_{3}-s_{2} s_{3}\right)\right]-0,022 s_{5}\left[s_{4}\left(c_{2} s_{3}+c_{3} s_{2}\right)-c_{4}\left(c_{2} c_{3}-s_{2} s_{3}\right)\right],
\end{aligned}
$$

where $c_{i}=\cos \theta_{i}, s_{i}=\sin \theta_{i}$. To solve the direct kinematics problem, the Denavit-Hartenberg parameters for the robot's thumb are given in Table 2 .

\begin{tabular}{|c|c|c|c|c|}
\hline № link & $\mathbf{d , ~} \mathbf{m}$ & $\mathbf{r , ~} \mathbf{m}$ & $\boldsymbol{\theta}$ & $\boldsymbol{\alpha}$ \\
\hline 1 & 0 & 0 & $\theta_{1}$ & 90 \\
\hline 2 & $-0,0425$ & 0,07 & $\theta_{2}$ & 0 \\
\hline 3 & 0 & 0,025 & 90 & 0 \\
\hline 4 & 0 & 0,038 & $\theta_{4}$ & 0 \\
\hline 5 & 0 & 0,028 & $\theta_{5}$ & 0 \\
\hline 6 & 0 & 0,022 & $\theta_{6}$ & 0 \\
\hline
\end{tabular}

Table 2. Denavit-Hartenberg parameters for the thumb

Next, building the transformation matrices for each of the links $A_{1}, \ldots A_{6}$, and the transformation matrix,. as shown above similarly we obtain formulas describing the position of the thumb in space.

\subsection{The solution of the inverse kinematics problem}

The inverse problem of kinematics consists in finding the angles between the links, at known coordinates of the end of the kinematic chain of links. With its help you can find the angles to which you need to turn the links in order, so that the end of the robot's finger is at the specified point. The problem considered in this paper differs from the usual one, since it operates with five finite dependent points, and not with one.

From the relations describing the position of the end of each of the robot's fingers, depending on the values of the angles $\theta$ obtained in solving the direct kinetic problem, we formulate a system of equations of the following form:

$$
p_{x y z}^{i}=f\left(\theta_{1}^{i}, \ldots, \theta_{n}^{i}\right),
$$


where $x y z$ - axis, the coordinate of which determines the equation, $i$ is the number of the finger whose coordinate is defined by equation, $n$ - number of links in this finger.

Since the angles $\theta_{1}$ and $\theta_{2}$ are the same for each of the fingers, and the angles responsible for rotation of the joints of the fingers are equal, then the system of non-linear equations can be written in the following form

$$
\left\{\begin{array}{l}
p_{x}^{5}=f_{x}^{5}\left(\theta_{1}, \theta_{2}, \theta_{3}^{5}\right), \\
p_{y}^{5}=f_{y}^{5}\left(\theta_{1}, \theta_{2}, \theta_{3}^{5}\right), \\
p_{z}^{5}=f_{z}^{5}\left(\theta_{1}, \theta_{2}, \theta_{3}^{5}\right), \\
p_{x}^{4}=f_{x}^{4}\left(\theta_{1}, \theta_{2}, \theta_{3}^{4}\right), \\
p_{y}^{4}=f_{y}^{4}\left(\theta_{1}, \theta_{2}, \theta_{3}^{4}\right), \\
p_{z}^{4}=f_{z}^{4}\left(\theta_{1}, \theta_{2}, \theta_{3}^{4}\right), \\
p_{x}^{3}=f_{x}^{3}\left(\theta_{1}, \theta_{2}, \theta_{3}^{3}\right), \\
p_{y}^{3}=f_{y}^{3}\left(\theta_{1}, \theta_{2}, \theta_{3}^{3}\right), \\
p_{z}^{3}=f_{z}^{3}\left(\theta_{1}, \theta_{2}, \theta_{3}^{3}\right), \\
p_{x}^{2}=f_{x}^{2}\left(\theta_{1}, \theta_{2}, \theta_{3}^{2}\right), \\
p_{y}^{2}=f_{y}^{2}\left(\theta_{1}, \theta_{2}, \theta_{3}^{2}\right), \\
p_{z}^{2}=f_{z}^{2}\left(\theta_{1}, \theta_{2}, \theta_{3}^{2}\right), \\
p_{x}^{1}=f_{x}^{1}\left(\theta_{1}, \theta_{2}, \alpha_{2}^{1}, \theta_{4}^{1}\right), \\
p_{y}^{1}=f_{y}^{1}\left(\theta_{1}, \theta_{2}, \alpha_{2}^{1}, \theta_{4}^{1}\right), \\
p_{z}^{1}=f_{x}^{1}\left(\theta_{1}, \theta_{2}, \alpha_{2}^{1}, \theta_{4}^{1}\right),
\end{array},\right.
$$

To solve the system, we use the function lsqnonlin package MATLAB. As a result of the simulation, we obtain matrices that store the position and orientation of the ends of the fingers of the robot's arm in space. Has been developed a program that processes the current set angles and depicts the position of the robot's fingers in 3D space (see fig. 5).

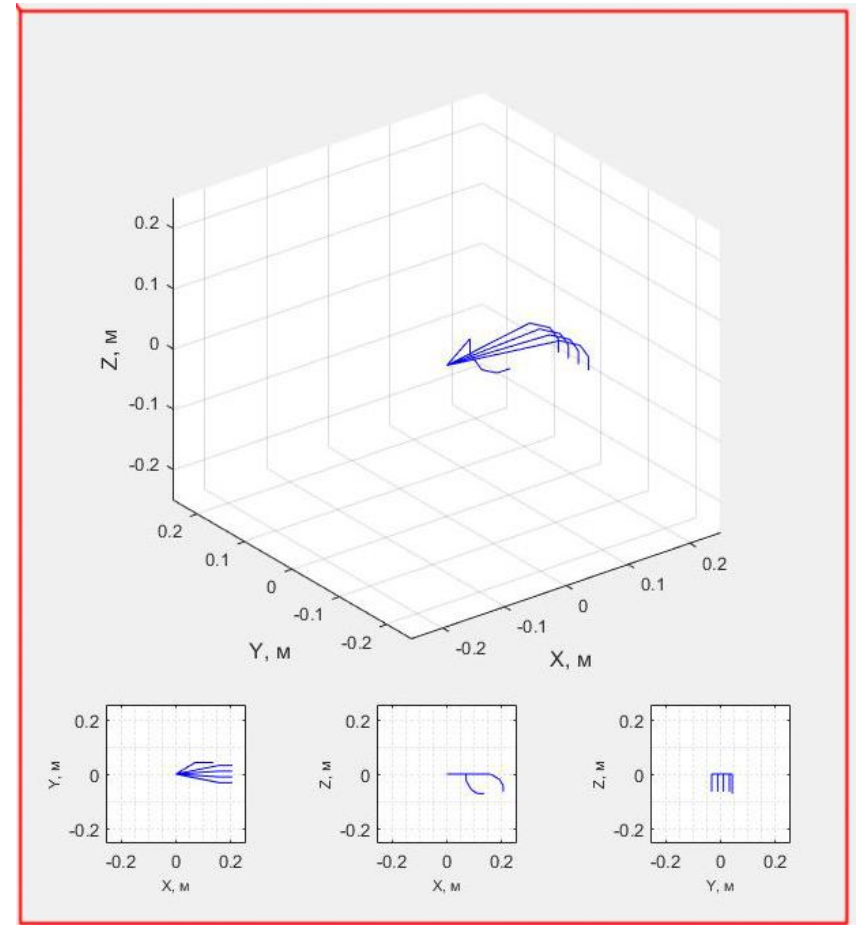

Fig. 5. Graphical display of the current position of the arm 


\section{Conclusion}

In this paper, a mathematical model of the robot brush SAR-400 was developed on the basis of the DenavitHartenberg method. Since, due to the peculiarities of the SAR-400 structure, some simplifications have been made, the mathematical model describes the robot's arm developed with some error. In the future, after describing the behavior of the fingers of the robot's arm with greater accuracy, the model used can be upgraded and the system will work with model of the robot's arm, which fully corresponds to the real.

Further research within the framework of the considered direction will be directed to refine the mathematical model for solving problems related to the fine motorics of manipulators. It is also planned to develop a specialized library of functions for controlling the robot manipulator SAR-400 in real time.

The obtained mathematical model of the robot's arm allows us to program a real sample, for example, through the Matlab interface and visually analyze the adequacy of the developed algorithms, testing them on industrial tasks. This will also allow to investigate the design features of the SAR-400 arm. The analytical approach to constructing the control laws for the robot's arm on the basis of the obtained mathematical model will become a good alternative and a test part when developing control laws based on the application of the neural network.

\section{Acknowledgments}

The reported study was funded by RFBR and Government of the Sevastopol according to the research project №1848-920022.

\section{References}

[1] Furuta, K.; Kosuge, K.; Mukai, N. (1988). Control of Articulated Robot Arm with Sensory Feedback: Laser Beam Tracking System. IEEE Trans. Ind. Electron., 35, 31-39, ISSN 0278-0046

[2] Munasinghe, S.R.; Nakamura, M.; Goto, S.; Kyura, N. (2001). Optimum Contouring of Industrial Robot Arms under Assigned Velocity and Torque Constraints. IEEE Trans. Syst. Man Cybern., Part C, 31, 159-167, ISSN 1094-6977

[3] Koga, M.; Kosuge, K.; Furuta, K.; Nosaki, K. (1992). Coordinated Motion Control of Robot Arms Based on the Virtual Internal Model. IEEE Trans. Robot. Autom., 8, 77-85, ISSN 1042-296X

[4] Efe, M.O. (2008). Fractional Fuzzy Adaptive Sliding-Mode Control of a 2-DOF Direct-Drive Robot Arm. IEEE Trans. Syst. Man Cybern. Part B, 38, 1561-1570, ISSN 1083-4419

[5] Huynh, H[oai] N[am]; Verlinden, O[livier] \& Riviere -Lorphevre, E[douard] (2017). Robotic Machining Simulation using a Simplified Multibody Model, Proceedings of the 28th DAAAM International Symposium, pp.0885-0894, B. Katalinic (Ed.), Published by DAAAM International, ISBN 978 -3-902734-11-2, ISSN 17269679, Vienna, Austria.

[6] Damic, V[jekoslav]; Cohodar, M[aida] \& Tvrtkovic, M[arko] (2016). Inverse Dynamic Analysis of Hobby Robot uArm by Matlab/Simulink, Proceedings of the 27th DAAAM International Symposium, pp.0095-0101,B. Katalinic (Ed.), Published by DAAAM International, ISBN 978-3-902734-08-2, ISSN 1726-9679, Vienna, Austria.

[7] J.Denavit, R.S.Hartenberg, (1955). A kinematic notation for lower-pair mechanisms based on matrices, Transactions ASME Journal of Applied Mechanics, 23, 215-221

[8] Spong, W. Mark, M.Vidyasagar (1989). Robot Dynamics and Control, John Wiley \& Sons, ISBN 9780471503521, New York

[9] P. I. Corke, (2007). A simple and systematic approach to assigning Denavit-Hartenberg parameters, IEEE Transactions on Robotics, vol. 23, pp. 590-594, ISSN 1552-3098 